\title{
EVOLUTION OF THE ROTATIONAL MOTION \\ OF A PLANET WITH A LIQUID CORE
}

\author{
V.V. SIDORENKO \\ Keldysh Institute of Applied Mathematics \\ Moscow, Russia
}

\begin{abstract}
Secular changes in the rotational motion of a planet due to dissipation of energy in its core are investigated. It is assumed that the effect of a liquid core is equivalent to the action on a "frozen" planet of a special type of nonconservative torque. The Bogoliuboff-Mitropolsky method is used to explain the secular effects. The following qualitative features of the evolution of the motion are established: the fast rotation of a planet gradually becomes slower and the inclination of its equator to the orbital plane gradually increases.
\end{abstract}

\section{Introduction and Basic Assumptions}

We examine the rotational motion of an axisymmetric planet with a liquid core in a central gravitational field. The planet's center of mass $O$ moves about an attracting center on a circular nonevolving orbit. The characteristic rotational velocity of the planet about its center of mass $\omega_{\text {rot }}$ substantially exceeds the orbital angular velocity $\omega_{o}: \omega_{o} / \omega_{\text {rot }}=\varepsilon \ll 1$. The planet's core is represented as a spherical cavity containing a highviscosity incompressible fluid. The assumption of high viscosity means that the Ekman number

$$
E=\frac{\nu}{\omega_{\text {rot }} R_{\text {core }}^{2}} \gg 1
$$

where $R_{\text {core }}$ is the cavity radius, and $\nu$ is the kinematic viscosity of the fluid. We further assume that $E \sim \varepsilon^{-n}, n \geq 5$.

The problem of the motion of a planet with a liquid core was studied by Sludski, Poincaré, and many other scientists. In particular, the secular effects due to the influence of the liquid core were examined by Aoki, Kubo, Neron de Surgy et al..

I. M. Wytrzyszczak, J. H. Lieske and R. A. Feldman (eds.),

Dynamics and Astrometry of Natural and Artificial Celestial Bodies, 313, 1997.

(C) 1997 Kluwer Academic Publishers. Printed in the Netherlands. 


\section{Motion of Matter in a Planetary Core}

In the system of coordinates $O x y z$, rigidly connected to the mantle, the motion of matter in the core is described by the Navier-Stokes equation

$$
\begin{gathered}
\frac{1}{\nu}\left[\frac{\partial \mathbf{u}}{\partial t}+(\mathbf{u} \nabla) \mathbf{u}+2 \omega \times \mathbf{u}+\frac{d \omega}{d t} \times \mathbf{r}\right]-\Delta \mathbf{u}+\nabla q=0, \\
q=\frac{1}{\nu}\left[\frac{p}{\rho}+U-\frac{1}{2}(\omega \times \mathbf{r})^{2}\right]
\end{gathered}
$$

Here, $\mathbf{r}$ is the position vector of an arbitrary point relative to the planet's center of mass; $\mathbf{u}(\mathbf{r}, t)$ and $p(\mathbf{r}, t)$ are the velocity and pressure fields; $U(\mathbf{r})$ is the gravitational and inertial potential due to the center of the mass' motion; $\rho$ is the core density; and $\omega$ is the rotational velocity of the trihedron $O x y z$.

Equation (1) is used in place of the continuity equation $\operatorname{div} \mathbf{u}=0$ and the kinematic condition $\left.\mathbf{u}\right|_{\Gamma}=0$, where $\Gamma$ is the core-mantle boundary. We choose the units of mass, length, and time such that $\rho=O(1), R_{\text {core }}=$ $O(1), \quad \nu=O(E)$, and $\omega_{o}=\varepsilon$.

For $E \gg 1$, the motion of the matter in the planet's core is of the nature of a quasistationary Stokes flow (Landau et al., 1986), which allows the inertial terms in (1) to be neglected. The velocity field satisfying the conditions

$$
\Delta \mathbf{u}=\nabla q+\frac{d \omega}{d t} \times \mathbf{r} / \nu, \quad \operatorname{div} \mathbf{u}=0,\left.\quad \mathbf{u}\right|_{\Gamma}=0
$$

has the form

$$
\mathbf{u}=\nu^{-1} \nabla f \times \frac{d \omega}{d t}
$$

where $f(\mathbf{r})=-\left(r^{2}-R_{\text {core }}^{2}\right)^{2} / 40$.

It follows from (2) that the motion of the core matter is subordinate to the motion relative to the center of mass. As shown by Chernous'ko (1968), this fact permits to consider the rotational motion of a planet with a liquid core as the motion of a "frozen" planet under the influence of a special type of torque

$$
\mathbf{M}_{c}=\kappa\left[\left(\frac{d^{2} \omega}{d t^{2}}\right)_{f r}+\omega \times\left(\frac{d \omega}{d t}\right)_{f r}\right], \quad \kappa=\frac{8 \pi \rho R_{c o r e}^{7}}{525 \nu}=O\left(E^{-1}\right)
$$

The derivatives $\left(d^{2} \omega / d t^{2}\right)_{f r}$ and $(d \omega / d t)_{f r}$ are calculated from the equations of motion for a frozen planet in a gravitational field. 


\section{Motion of the Planet Relative to the Center of Mass}

To describe the rotational motion of a planet we will use the canonical variables of Andoyer (Deprit, 1967):

$$
L, G, H, l, g, h \text {. }
$$

Here $L$ is the projection of the angular momentum $\mathbf{G}$ on the planet's figure axis, and $H$ is the projection of the angular momentum on the normal to the orbital plane. The equations of motion in the Andoyer variables are

$$
\begin{aligned}
\frac{d(L, G, H)}{d t} & =-\frac{\partial K}{\partial(l, g, h)}+f_{(L, G, H)}, \\
\frac{d(l, g, h)}{d t} & =\frac{\partial K}{\partial(L, G, H)}+f_{(l, g, h)} .
\end{aligned}
$$

The function $K$ in (3) is the Hamiltonian of the "frozen" planet in the central gravitational field. It can be written as follows:

$$
K(L, G, H, l, g, h, t)=\frac{1}{2}\left[\left(\frac{1}{C}-\frac{1}{A}\right) L^{2}+\frac{G^{2}}{A}\right]+\frac{3}{2} \varepsilon^{2}(C-A)(\mathbf{s}, \mathbf{e})^{2} .
$$

Here $A$ and $C$ are the equatorial and axial moments of inertia of the planet $(A<C)$; e is the unit vector of the planet's figure axis; $\mathbf{s}=\mathbf{R} /|\mathbf{R}|$, where $\mathbf{R}$ is the vector from $O$ to the attractive center. The scalar product is

$(\mathbf{s}, \mathbf{e})=\sin (h-\varepsilon t) \sin I \cos J+[\cos (h-\varepsilon t) \sin g+\sin (h-\varepsilon t) \cos g \cos I] \sin J$,

where $I$ is the angle between the angular momentum vector $\mathbf{G}$ and normal to the orbital plane, and $J$ is the angle between $\mathbf{G}$ and the planet's figure axis.

Functions $f_{L}, f_{G}, f_{H}, f_{l}, f_{g}$, and $f_{h}$ characterize the influence of the nonconservative torque $\mathbf{M}_{c}$ on the rotational motion of the planet.

The evolution of the motion can be separated into two stages with different timescales. In the first stage (stage of fast dissipative evolution)

$$
G \approx G(0), \quad H \approx H(0), \quad L \rightarrow G \operatorname{sign} L(0) .
$$

We assume that $L \rightarrow G(L(0)>0)$. The change in the angle between the figure axis and $G$ obeys the law

$$
J(t)=\operatorname{arctg}\left(e^{-\sigma t} \operatorname{tg} J(0)\right), \quad \sigma=\kappa\left(\frac{1}{A}-\frac{1}{C}\right) \frac{G^{2}(0)}{A^{2}} .
$$

The characteristic timescale for fast dissipative evolution $T_{\text {fast }} \sim E$. 
After completion of the fast dissipative evolution phase the planet's figure axis will precess around the normal to the orbital plane. This is the stage of slow dissipative evolution.

Since for $J \rightarrow 0$ a singularity occurs in Eqs.(3), it is convenient to introduce canonical variables $\tilde{p}, \widetilde{G}, \widetilde{H}, \tilde{q}, \widetilde{g}, \tilde{h}$ guaranteeing regularity of the right-hand sides of the equations of motion. These variables are connected to the Andoyer variables by the relations

$$
\begin{gathered}
\tilde{p}=\sqrt{2(G-L)} \cos l, \quad \tilde{G}=G, \quad \tilde{H}=H, \\
\tilde{q}=-\sqrt{2(G-L)} \sin l, \quad \tilde{g}=g+l, \quad \tilde{h}=h-\varepsilon t .
\end{gathered}
$$

Taking into account, that in the stage of slow dissipative evolution $\tilde{p}=$ $O\left(\varepsilon^{2}\right), \quad \tilde{q}=O\left(\varepsilon^{2}\right)$, we perform a noncanonical stretching substitution of variables

$$
P=\varepsilon^{-2} \tilde{p}, \quad Q=\varepsilon^{-2} \tilde{q} .
$$

The resulting equations of motion can be written as

$$
\frac{d \Xi}{d t}=\sum_{k=0}^{\infty} \varepsilon^{k} V_{k}(\Xi)+\kappa \sum_{k=0}^{\infty} \varepsilon^{k} W_{k}(\Xi),
$$

where $\Xi=(P, \widetilde{G}, \widetilde{H}, Q, \tilde{g}, \tilde{h})$.

For an approximate description of the slow dissipative evolution, we will use the formal solution of equations (4) (Bogoliuboff et al., 1961):

$$
\begin{gathered}
P=\sum_{k=0}^{\infty} \varepsilon^{k} u_{k}(\bar{G}, \bar{H}, \bar{g}, \bar{h}), \quad Q=\sum_{k=0}^{\infty} \varepsilon^{k} v_{k}(\bar{G}, \bar{H}, \bar{g}, \bar{h}), \\
\tilde{G}=\bar{G}+\sum_{k=1}^{\infty} \varepsilon^{k} N_{G k}(\bar{G}, \bar{H}, \bar{g}, \bar{h}), \quad \tilde{H}=\bar{H}+\sum_{k=1}^{\infty} \varepsilon^{k} N_{H k}(\bar{G}, \bar{H}, \bar{g}, \bar{h}), \\
\tilde{g}=\bar{g}+\sum_{k=1}^{\infty} \varepsilon^{k} M_{g k}(\bar{G}, \bar{H}, \bar{g}, \bar{h}), \quad \tilde{h}=\bar{h}+\sum_{k=1}^{\infty} \varepsilon^{k} M_{g k}(\bar{G}, \bar{H}, \bar{g}, \bar{h}),
\end{gathered}
$$

where the functions $u_{k_{1}}, N_{G k_{2}}, N_{H k_{2}}, v_{k_{1}}, M_{g k_{2}}, M_{h k_{2}}, \quad\left(k_{1}=0,1, \ldots\right.$; $k_{2}=1,2, \ldots$ ) are periodic in both $\bar{g}$ and $\bar{h}$ with period $2 \pi$, and $\bar{G}, \bar{H}, \bar{g}$, and $\bar{h}$ are functions of time satisfying the differential equations

$$
\frac{d \bar{G}}{d t}=\kappa \sum_{k=0}^{\infty} \varepsilon^{k} A_{G k}(\bar{G}, \bar{H}), \quad \frac{d \bar{H}}{d t}=\kappa \sum_{k=0}^{\infty} \varepsilon^{k} A_{H k}(\bar{G}, \bar{H}),
$$




$$
\frac{d \bar{g}}{d t}=\bar{G} / C+\sum_{k=1}^{\infty} \varepsilon^{k} B_{g k}(\bar{G}, \bar{H}), \quad \frac{d \bar{h}}{d t}=-\varepsilon+\sum_{k=2}^{\infty} \varepsilon^{k} B_{h k}(\bar{G}, \bar{H}) .
$$

After elementary operations we find:

$$
\begin{gathered}
A_{G 0}=A_{H 0}=A_{G 1}=A_{H 1}=A_{G 2}=A_{H 2}=A_{G 3}=A_{H 3} \equiv 0, \\
A_{G 4}(\bar{G}, \bar{H})=-\frac{9(C-A)^{2}}{8 \bar{G} C} \sin ^{2} \bar{I}\left(4-3 \sin ^{2} \bar{I}\right), \\
A_{H 4}(\bar{G}, \bar{H})=-\frac{9(C-A)^{2}}{2 \bar{G} C} \sin ^{2} \bar{I} \cos \bar{I}
\end{gathered}
$$

Here $\bar{I}=\arccos \bar{H} / \bar{G}$. To precise $O(\varepsilon), \bar{I}$ characterizes the angle between the planet's figure axis and the normal to the orbital plane during the slow dissipative evolution stage.

\section{Secular Changes in the Motion of a Planet}

For a qualitative analysis of the secular effects, we examine the reduced system

$$
\frac{d \bar{G}}{d t}=\varepsilon^{4} \kappa A_{G 4}(\bar{G}, \bar{H}), \quad \frac{d \bar{H}}{d t}=\varepsilon^{4} \kappa A_{H 4}(\bar{G}, \bar{H}) .
$$

Using (5), the following result can be obtained:

$$
\begin{gathered}
\sqrt{G^{2}(0)-2 \chi d_{1} t} \leq \bar{G}(t) \leq \sqrt{G^{2}(0)-2 \chi d_{2} t} \\
\left(1-\frac{2 \chi d_{1} t}{G^{2}(0)}\right)^{\frac{3}{8 d_{1}}} \leq \frac{\cos \bar{I}(t)}{\cos I(0)} \leq\left(1-\frac{2 \chi d_{2} t}{G^{2}(0)}\right)^{\frac{3 \sin ^{4} I(0)}{8 d_{2}}} .
\end{gathered}
$$

Here

$d_{1}=\frac{1}{3}, \quad d_{2}=\min \left\{\frac{1}{4}, \sin ^{2} I(0)\left[1-\frac{3}{4} \sin ^{2} I(0)\right]\right\}$ in the case $|\sin I(0)| \leq$ $\sqrt{2 / 3}$ and $d_{1}=\sin ^{2} I(0)\left[1-\frac{3}{4} \sin ^{2} I(0)\right], \quad d_{2}=\frac{1}{4}$ in the case $|\sin I(0)|>$ $\sqrt{2 / 3}, \chi=9 \varepsilon^{4} \kappa(C-A)^{2} / 2 C$.

Thus, the rotation of a planet with a liquid core gradually becomes slower, and the inclination of its equator to the orbital plane gradually increases.

The final stage of evolution of the rotation was studied numerically. As it turns out, a sharp increase in the angle between the figure axis and the angular momentum vector occurs, when the angular velocity of rotation of the planet exceeds the velocity of its orbital motion $\omega_{0}$ by two times. Subsequently, the cone around the normal to the orbital plane enclosing 
the motion of the figure axis gradually constricts. The limiting motion of the planet is a prograde rotation about the normal to the orbital plane with angular velocity $\approx 2 \omega_{0}$.

\section{References}

Aoki, S.: 1969, "Friction between the mantle and core of the Earth as a cause of the secular change in obliquity", Astron. J. 74, 284-291.

Bogoliuboff, N.N. and Mitropolsky, Yu.A.: 1961, Asymptotic Methods in the Theory of Nonlinear Oscillations, Gordon Breach, New York.

Chernous'ko, F.L.: 1968, Motion of a Rigid Body with Cavities Containing Viscous Fluid, VTs AN SSSR, Moscow (in Russian).

Deprit, A.: 1967, "Free rotation of a rigid body studied in the phase plane", Am. J. Phys. 35, 424-428.

Kubo, Y.: 1979, "A core-mantle interaction in the rotation of the Earth", Celest. Mech. $19,215-241$.

Landau, L. and Lifshitz, E.M.: 1986, Hydrodynamics, Nauka, Moscow (in Russian).

Neron de Surgy, O. and Laskar, J.: 1995, "On the long term evolution of the spin of the Earth", Astron. Astrophys., accepted for publication.

Poincaré, H.: 1910, "Sur la précession des corps déformables", Bull. Astron. 27, 321-356.

Sludski, F.A.: 1896, "De la rotation de la Terre supposée fluide à l'intérieur", Bull. Soc. Imp. Natur., 258-318. 